\title{
Recognition-controlled membrane translocation for signal transduction across lipid bilayers
}

\author{
Matthew J. Langton, ${ }^{a *}$ Nicholas H. Williams ${ }^{b}$ and Christopher A. Hunter ${ }^{a}$ \\ a Department of Chemistry, University of Cambridge, Lensfield Road, Cambridge CB2 1EW, United Kingdom \\ ${ }^{b}$ Department of Chemistry, University of Sheffield, Sheffield S3 7HF, United Kingdom \\ KEYWORDS Signal transduction; Molecular recognition; Bilayer membranes; Vesicles; Molecular motion; Switchable catalyst.
}

\begin{abstract}
Membrane signaling proteins transduce information across lipid bilayer membranes in response to extra-cellular binding of chemical messengers. The design of chemical systems that initiate transmembrane signal transduction through molecular binding events is a critical step towards preparing responsive synthetic vesicles. Here we report a vesicle-based signaling system controlled by a metal cation binding event. Competition between binding of copper ions to a membrane-embedded synthetic transducer and to an extra-vesicle messenger (EDTA) is used to control translocation of the transducer across the lipid bilayer. The translocation process is coupled to activation of a catalyst that turns over of encapsulated substrates on the inside of the vesicle to generate an amplified fluorescence output signal. External EDTA and copper ions can be used to reversibly switch catalysis inside the vesicles on and off in a controlled manner.
\end{abstract}

\section{INTRODUCTION}

Membrane signaling proteins transduce chemical information across lipid bilayer membranes in response to extra-cellular binding of small molecule messengers. ${ }^{1}$ Recognition of these "first messenger" signals leads to a structural change in transmembrane receptor proteins, leading to an intracellular response. Typically this response is enhanced by a subsequent cascade of enzymatic reactions, generating multiple copies of a "second messenger", leading to amplification of the signal. First messenger binding induces transmembrane signal transduction through one of two mechanisms in natural systems: dimerization of the membrane spanning receptors (e.g. tyrosine kinase receptors ${ }^{2}$ ) or through a global conformational change (e.g. G-coupled receptors ${ }^{3}$ ). Mimicking these processes in synthetic systems has proven extremely challenging, but there are numerous anticipated applications in drug delivery, sensing and synthetic proto-cells. Whilst many systems have been reported which can transport chemical signals such as ions and small molecules across lipid bilayer membranes, ${ }^{4-10}$ those that transduce information without physical transport of matter are rare ${ }^{11-16}$ Recently, we demonstrated a novel mechanism for signal transduction and amplification across lipid bilayer membranes, which is not observed in Nature. ${ }^{17}$ This mechanism uses an input chemical signal to control the translocation of a synthetic membrane-embedded transducer molecule from the outer to the inner leaflet of a vesicle lipid bilayer membrane (Fig. 1). This translocation process exposes a catalyst to the internal aqueous solution of the vesicle, which turns over encapsulated substrate molecules leading to generation of an amplified output signal.

In the first-generation system, an extra-vesicle $\mathrm{pH}$ change was used as the input signal to initiate membrane translocation of a synthetic signal transducer, by deprotonating an ammonium head group exposed to the external solution. Here we demonstrate that a molecular recognition event can be used to initiate membrane translocation, representing a crucial step towards designing artificial signaling systems able to respond to diverse targets through supramolecular binding events.

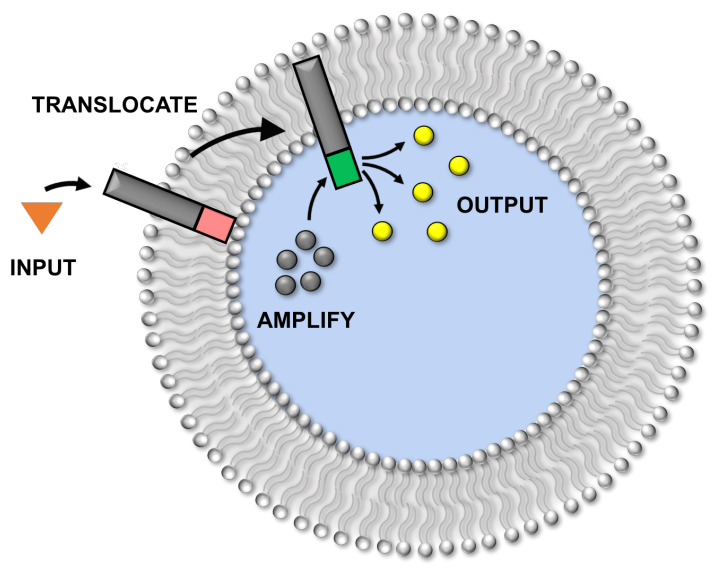

Figure 1. Transmembrane signal transduction and amplification using controlled translocation of a membrane-embedded transducer. An external input signal (orange) initiates the translocation of a synthetic molecule from the outer to the inner leaflet of a lipid bilayer membrane. When in the outer leaflet, the catalytic head group of the molecule is embedded in the bilayer and inactive (red). In the inner leaflet, the catalyst is exposed to the inner aqueous solution of the vesicle where it is active (green), and catalyzes the turn-over of a large number of substrate molecules to generate the amplified output signal inside the vesicle. 
(a)

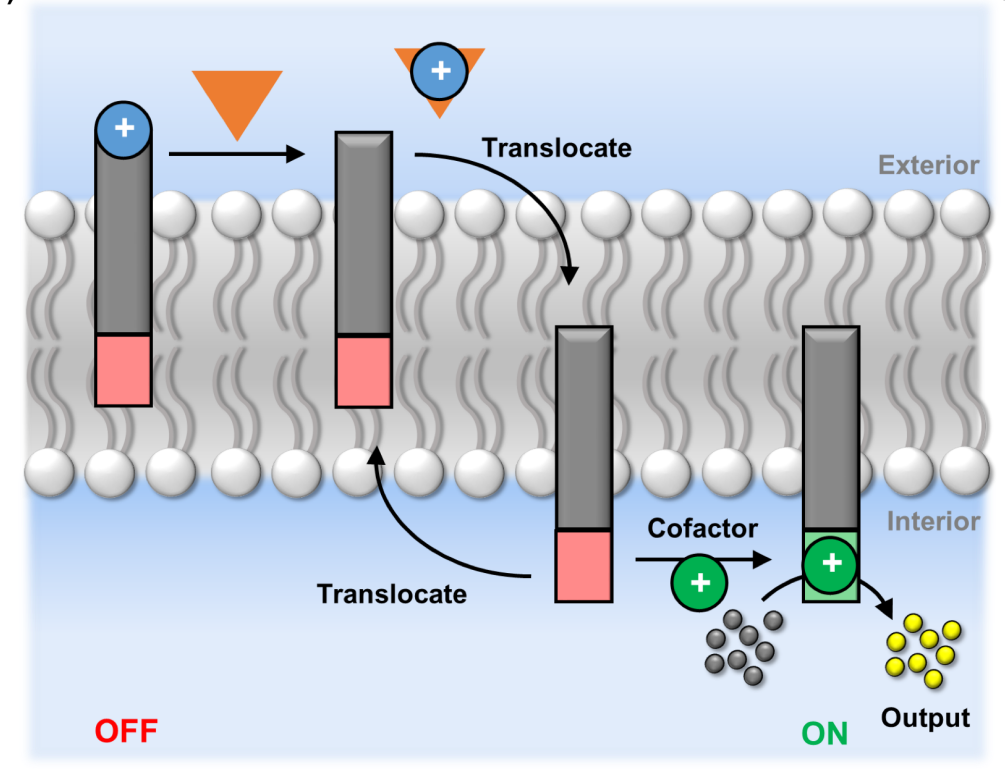

(b)

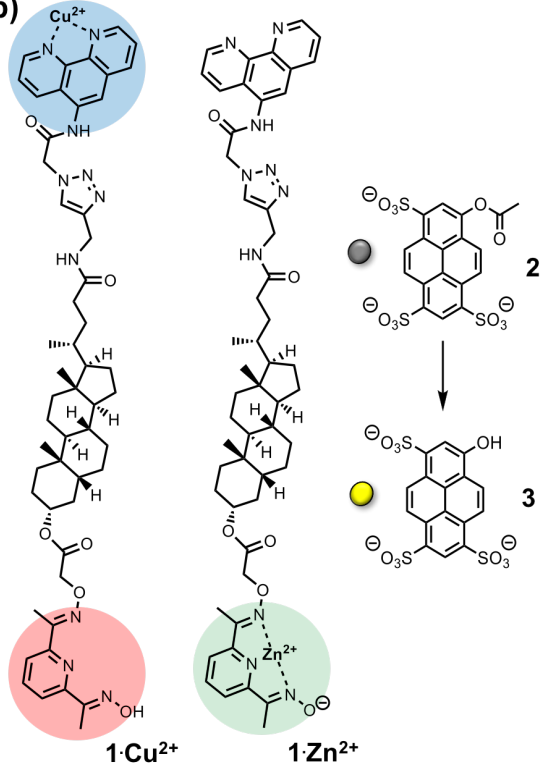

Figure 2. (a) Schematic representation of a supramolecular signal transduction system. The input signal (orange) displaces the bound cation (blue) from the external head group of the transducer, which neutralizes the head group and allows the transducer to translocate through the membrane. Subsequent binding of a metal cation cofactor from the internal solution of the vesicle to the catalytic head group (red) activates the catalyst (green), which generates the output signal (yellow) by turning over the encapsulated substrate. (b) Molecular structures of transducer 1 as the Cu(II) complex $\mathbf{1} \cdot \mathbf{C u}^{2+}$ (OFF state), the $\mathrm{Zn}(\mathrm{II})$-activated catalyst $\mathbf{1} \cdot \mathbf{Z n}^{2+}$ (ON state), substrate $\mathbf{2}$ and fluorophore product $\mathbf{3}$.

\section{APPROACH}

The approach to controlling membrane translocation of a synthetic signaling molecule through host-guest interactions is shown in Fig. 2a. The key is the differing solubility characteristics of polar and apolar head groups at lipid bilayer membrane interfaces: charged, polar moieties are preferentially located in the aqueous phase, whilst neutral, apolar moieties can enter the interior of the hydrophobic bilayer. Thus a chemical input signal that reverses the polarities of the head groups of a linear, bi-functional transducer molecule embedded in a membrane provides a mechanism for controlling translocation of the transducer from one leaflet of the bilayer to the other.

In the initial state of the system where the catalyst head group (red) is inactive (the OFF state), a charged guest is bound to the recognition head group (blue) holding it in the aqueous phase. Addition of the input signal (orange), which competes for guest binding, displaces the charged guest and neutralizes the recognition head group. This head group is thereby switched from a polar state to a membrane permeable apolar state, allowing the transducer to translocate across the bilayer from the outer to the inner leaflet. Here, the apolar catalyst head group binds a charged cofactor from the internal aqueous solution, switching this head group to a polar state (green) and locking the transducer in the ON state in the inner leaflet. Cofactor binding simultaneously activates the catalyst, which turns over multiple encapsulated substrate molecules to generate an amplified output signal (yellow).

To demonstrate this concept, we have designed a supramolecular signal transducer $\mathbf{1}$, which features a phenanthroline head group that can form strong host-guest interactions with transition metal cations. The structure of transducer 1 is depicted in Fig. $2 \mathrm{~b}$. The bifunctional transducer is comprised of two head groups linked by a hydrophobic steroid core, which serves to anchor it in the membrane. The outer recognition head group consists of a phenanthroline motif, which acts as a receptor for $\mathrm{Cu}$ (II) ions. When copper is bound, the head group is charged and held out of the membrane in the aqueous phase. Displacement of the bound cation reveals the neutral phenanthroline head group, which is soluble in the membrane and free to move through the bilayer. The other head group is a neutral pyridine-oxime ligand. Binding of $\mathrm{Zn}$ (II) cations from the internal solution of the vesicles switches this head group to a charged state and traps it in the aqueous phase, locking the transducer in the inner leaflet. Zinc binding acts to lower the $\mathrm{p} K_{\mathrm{a}}$ of the oxime from 11 to below 6 , and the oximate anion formed at neutral pH can act as a nucleophilic catalyst for ester hydrolysis. ${ }^{18}$ The ester substrate for this reaction, 2 , is highly charged and membrane impermeable. ${ }^{19}$ The product of the hydrolysis, 3, is fluorescent, providing a convenient spectroscopic handle with which to probe signaling events. ${ }^{20}$ In this design, the degree of signal amplification, [output]/[input], is simply determined by the concentration of substrate dissolved in the inner solution. In principle, the activation of just one transducer will lead to the turn-over of all encapsulated substrate molecules in a vesicle.

\section{RESULTS AND DISCUSSION}

Transducer 1 was synthesized from lithocholic acid as shown in Scheme 1. Alkyne-derivative 6 was synthesized from lithocholic acid by $N$-(3-dimethylaminopropyl)- $N$-ethylcarbodiimide hydrochloride (EDC) mediated amide coupling with propargyl amine, followed by condensation with chloroacetyl chloride. 6 was then coupled to phenanthroline azide 5 using a $\mathrm{Cu}(\mathrm{I})$-catalyzed alkyneazide click reaction, in the presence of tris[(1-benzyl-1H-1,2,3triazol-4-yl)methyl]amine (TBTA) to afford 7. The product was subsequently functionalized with the pyridine dioxime ligand by 
substitution with $\mathbf{8}$, followed by condensation with one equivalent of hydroxylamine in the presence of catalytic sodium acetate to afford transducer $\mathbf{1}$. Ester substrate $\mathbf{2}$ was prepared in one step from commercially available 8-hydroxypyrene-1,3,6-trisulfonic acid (HPTS) $3 .^{21}$

\section{Scheme 1. Synthesis of phenanthroline transducer 1}

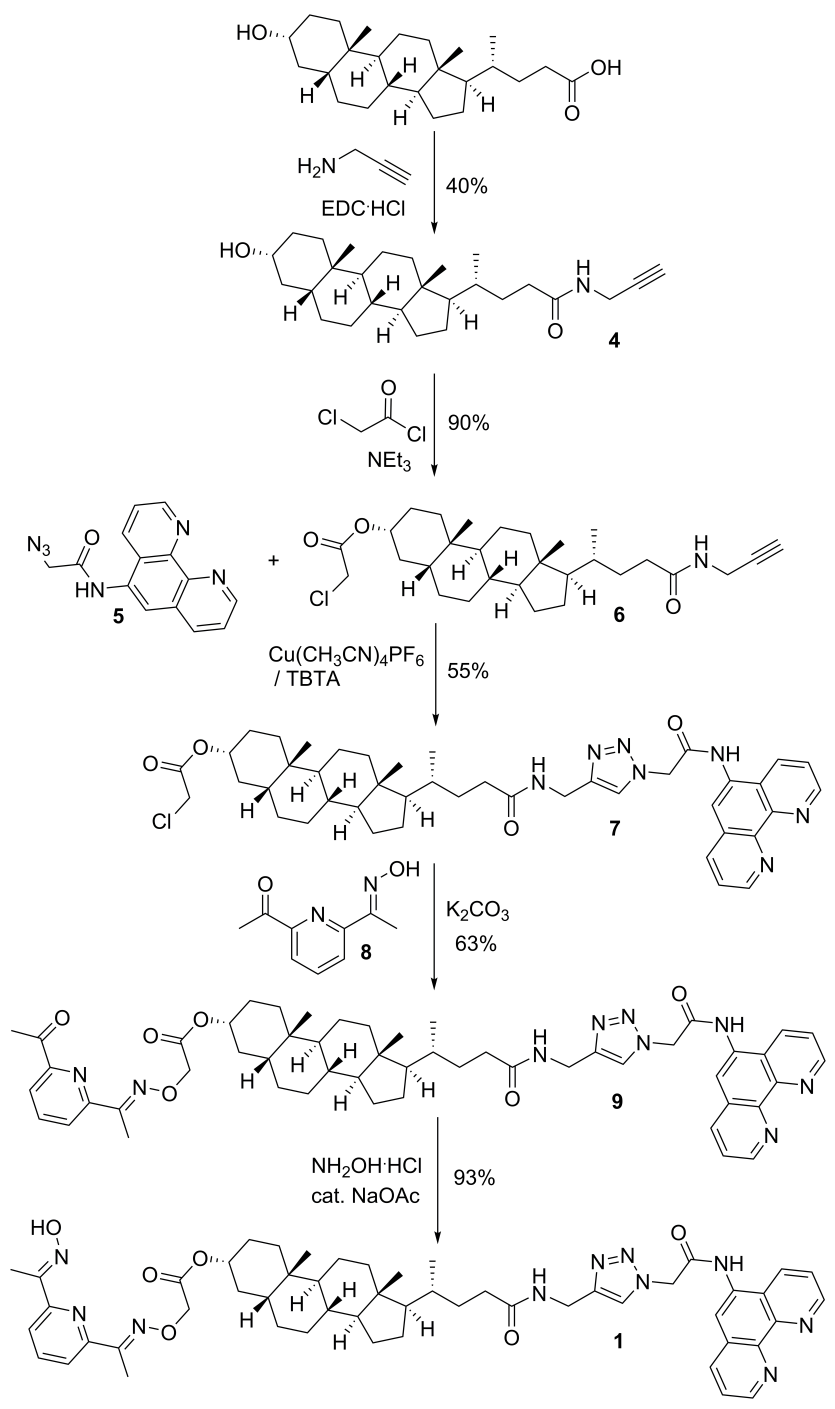

The initial OFF state of the system is obtained when the phenanthroline head group is bound to copper. To prepare the copper complex, $1 \cdot \mathrm{Cu}^{2+}, 1$ equivalent of $\mathrm{CuCl}_{2}$ was added to an ethanolic solution of 1,2-dioleoyl-sn-glycero-3-phosphocholine (DOPC) and 1,2-dioleoyl-sn-glycero-3-phosphoethanolamine (DOPE) lipids and transducer 1 ( $1 \mathrm{~mol} \%$ loading), and the mixture was then dried under vacuum. The film of lipids and transducer was rehydrated at $\mathrm{pH} 7$ in HEPES buffer containing $250 \mu \mathrm{M} \mathrm{ZnCl} 2$ and $250 \mu \mathrm{M}$ ester 2 by sonication. The suspension was extruded ${ }^{23}$ through a polycarbonate membrane to afford vesicles of an average diameter of around $200 \mathrm{~nm}$ (confirmed by DLS measurements) and final bulk concentrations of $1 \mathrm{mM}$ lipids and $10 \mu \mathrm{M}$ transducer complex $1 \cdot \mathrm{Cu}^{2+}$ (see ESI). A further sample of vesicles were prepared in an identical manner, but without the transducer complex 1. $\mathrm{Cu}^{2+}$ for the purposes of control experiments.

Both the phenanthroline and pyridine oxime head groups of transducer 1 can act as ligands for the binding of metal cations.
Under the vesicle preparation conditions, membrane-embedded 1 is exposed to both $\mathrm{Cu}$ (II) and $\mathrm{Zn}$ (II) cations in solution. The four possible 1:1 complexes that could be formed between the two metal cations and the two head groups are depicted in Fig. 3. The bar chart shows the estimated population of each complex for the con-

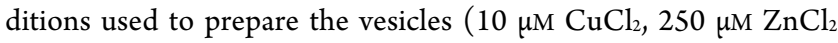
and $10 \mu \mathrm{M} \mathrm{1}$ ) using literature association constants for the corresponding phenanthroline ${ }^{22}$ (Phen) and 2,6-diacetylpyridine dioxime $^{18}$ (PyO) complexes. The copper-phenanthroline complex is the dominant species (>95\%), and this species represents the OFF state, so competition with the other possible complexes should not significantly affect assembly of the signaling system. ${ }^{24}$

The vesicle assembly procedure affords vesicles in which transducer complex $\mathbf{1} \cdot \mathrm{Cu}^{2+}$ is equally distributed between the outer and inner leaflets of the membrane. Therefore $50 \%$ of the transducers are in the correct orientation for signaling with the copperphenanthroline head group exposed to the external solution. Only these transducers are competent to catalyze hydrolysis of the encapsulated ester substrate in response to EDTA addition. EDTA is highly charged and does not penetrate lipid bilayer membranes, so does not prevent the internal $\mathrm{Zn}$ (II) cations forming the active complex.

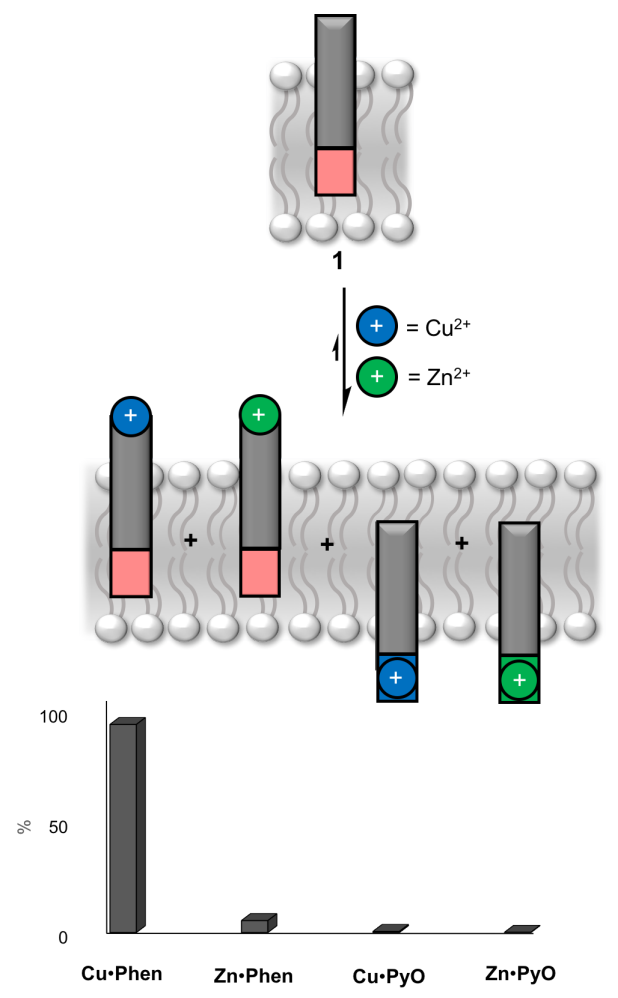

Figure 3. Metal complexes formed during the preparation of vesicles containing transducer 1 in the presence of $\mathrm{Cu}$ (II) and $\mathrm{Zn}$ (II) cations. The bar chart represents the estimated populations of each head group complex in the presence of $\mathrm{CuCl}_{2}(10 \mu \mathrm{M})$ and $\mathrm{ZnCl}_{2}(250 \mu \mathrm{M})$ and 1 $(10 \mu \mathrm{M})$.

Signaling experiments were conducted by adding an aliquot of aqueous EDTA solution to a suspension of vesicles in a cuvette, and measuring the fluorescence emission arising from the formation of 3 inside the vesicles. The results of a representative signaling experiment are shown in Fig. 4. In the control system, comprising of vesicles containing 2 and $\mathrm{ZnCl}_{2}$ but without the trans- 
ducer complex, there is only a very slight increase in fluorescence emission intensity over the timescale of the experiment, reflecting slow solvolysis of the substrate (blue data). This background reaction is unchanged by addition of EDTA to the extra-vesicular solution (purple data). For vesicles containing the transducer complex 1. $\mathrm{Cu}^{2+}$, in the absence of EDTA the behavior is similar to the control system, indicating that the signaling system is in the OFF state (red data). Addition of EDTA to the vesicle suspension led to a sharp increase in fluorescence intensity (green data) due to translocation of 1 across the bilayer. Formation of the zinc-pyridine oxime complex $1 \cdot \mathrm{Zn}^{2+}$ inside the vesicles generates the $\mathrm{ON}$ state leading to catalysis of the formation of $\mathbf{3}$ inside the vesicles. The compartmentalization of the ester hydrolysis reaction inside the vesicles, i.e. without leakage of either $\mathbf{2}$ or $\mathbf{3}$, was demonstrated by quenching experiments using a membrane-impermeable cationic quencher $p$-xylene-bis-pyridinium bromide (see Fig. S2). ${ }^{25}$ The rate of translocation of the transducer is fast relative to that of the catalyzed ester hydrolysis reaction, as there is no observable induction period in the hydrolysis kinetics which would be indicative of slow translocation and catalyst activation. The extent of amplification exhibited by the system is determined by the molar ratio of 3 produced inside the vesicles to EDTA added on the exterior. In these experiments, a 5-fold amplification of signal was achieved.

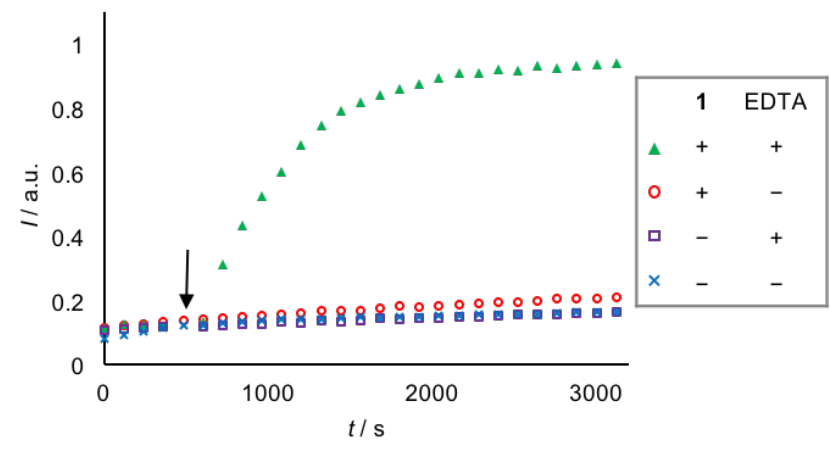

Figure 4. Time dependence of the normalized fluorescence emission intensity at $510 \mathrm{~nm}$ (exciting at $415 \mathrm{~nm}$ ) of vesicles in the presence $(+)$ and absence $(-)$ of membrane-embedded transducer $1 . \mathrm{Cu}^{2+}(10 \mu \mathrm{M})$ and following external EDTA addition $(5 \mu \mathrm{M})$. All experiments were conducted in $200 \mathrm{~nm}$ DOPC/DOPE vesicles ( $1 \mathrm{mM}$ final lipid concentration) containing $250 \mu \mathrm{M}$ ester 2, $250 \mu \mathrm{M} \mathrm{ZnCl}_{2}$ and $100 \mathrm{mM}$ HEPES buffer at $\mathrm{pH} 7$.

Fig. 5 shows that the fraction of transducer molecules that are in the ON state is the outcome of competing equilibria on the exterior and interior of the vesicles. The behavior of the system is governed by the association constants for these equilibria, which can be estimated from literature aqueous phase values for the copperphenanthroline complex $(\log K=9.0)$ and the zinc-2,6diacetylpyridine dioxime complex $(\log K=4.6)$. In the absence of EDTA (Fig. 5, top), the overall equilibrium lies in favor of the copper-phenanthroline complex, because the concentration of zinc inside the vesicles is only 50 times larger than the concentration of copper outside. However, when EDTA is added the copper is no longer available to bind the phenanthroline head group, because the association constant for the copper-EDTA complex is nine orders of magnitude larger. ${ }^{26}$ Under these conditions, the overall equilibrium lies in favor of the zinc-pyridine oxime complex (Fig. 5, bottom).
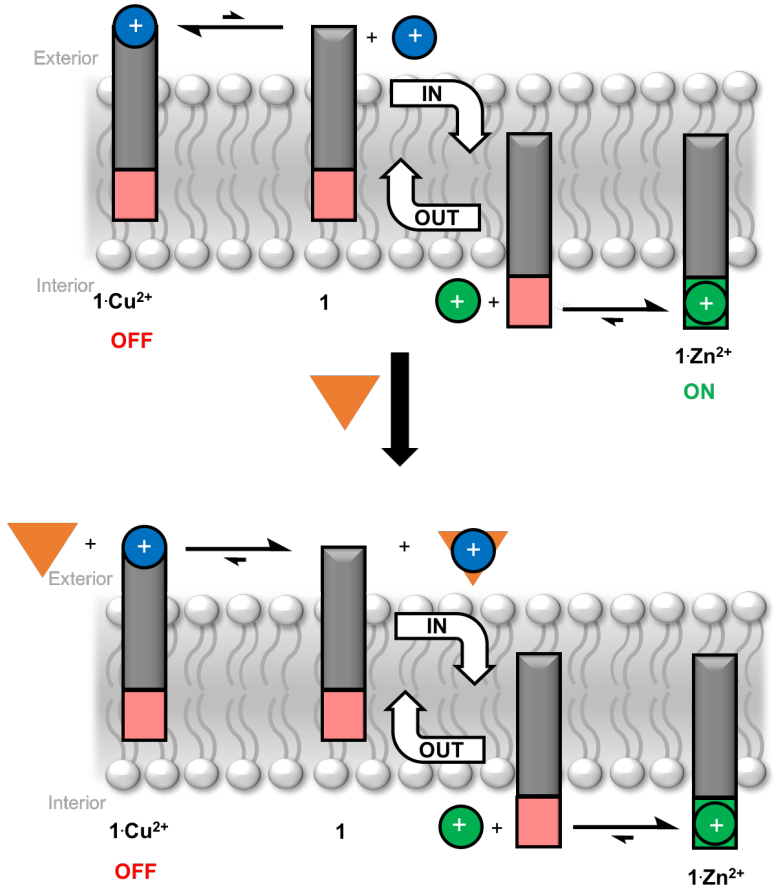

ON

Figure 5. Metal cation binding equilibria in the absence (top) and presence (bottom) of externally added EDTA (orange triangle).

Fig. 5 suggests that the concentration of zinc should be an important factor that affects signaling efficiency. In the presence of EDTA, the fraction of transducers that are in the ON state depends on the position of the equilibrium for zinc binding on the inside of the vesicles. Fig. 6 shows the outcome of variation in the concentration of zinc inside the vesicles. In the absence of internal zinc ions, the transducer complex has no effect on the rate of formation of 3 when EDTA is added to the external vesicle solution. In the presence of $25 \mu \mathrm{M}$ zinc ions, signal transduction is observed, and the rate of hydrolysis of $\mathbf{2}$ is increased on addition of EDTA to the external vesicle solution. When the concentration of zinc ions is increased 10-fold to $250 \mu \mathrm{M}$, the rate of hydrolysis of 2 increases further, indicating that more catalyst is present in the ON state, because more of the transducer is bound to zinc. Thus the concentration of zinc inside the vesicles can be used to modulate the proportion of transducers that are present in the $\mathrm{ON}$ state.

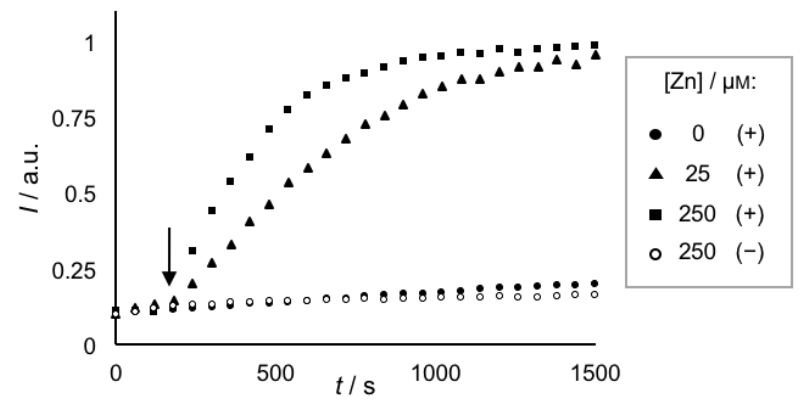

Figure 6. Time dependence of the normalized fluorescence emission intensity at $510 \mathrm{~nm}$ (exciting at $415 \mathrm{~nm}$ ) of vesicles containing varying amount of $\mathrm{ZnCl}_{2}$ in the internal solution, and in the presence $(+)$ or absence (-) of transducer complex $\mathbf{1} \cdot \mathbf{C u}^{2+}$. Black arrow denotes EDTA addition $(5 \mu \mathrm{M})$. 


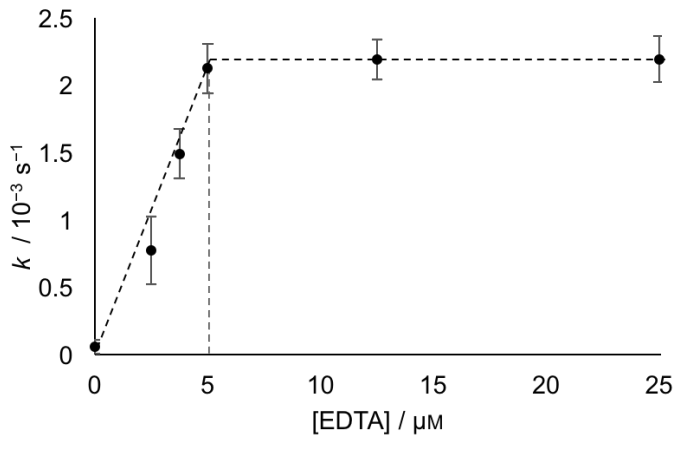

Figure 7. Relationship between the pseudo-first order rate constant, $k$, for the hydrolysis of vesicle encapsulated 2 and the external EDTA concentration. All experiments were conducted in $200 \mathrm{~nm}$ DOPC/DOPE vesicles $(1 \mathrm{~mm}$ final lipid concentration and $10 \mu \mathrm{M}$

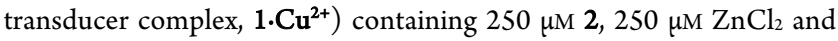
$100 \mathrm{mM}$ HEPES buffer at $\mathrm{pH} 7$. Error bars denote $2 \sigma$ obtained from a minimum of two repetitions.

Similarly, the proportion of transducers that are in the ON state should be related to the amount of EDTA that is added to the external vesicle solution. The concentration of active $\mathbf{1} \cdot \mathbf{Z n}^{2+}$ complex present in the ON state can be quantified using the rate constant for the hydrolysis of $\mathbf{2}$. The observed rate of ester hydrolysis inside the vesicles was therefore measured as a function external EDTA concentration (Fig. 7). Aliquots of an EDTA stock solution were added to separate identical vesicle samples containing the transducer complex $1 \cdot \mathrm{Cu}^{2+}$, and the change in the fluorescence emission intensity was fit to a pseudo-first order rate equation (see Fig. S3). The rate constant increases with increasing EDTA concentration until a maximum rate is reached at $5 \mu \mathrm{M}$ EDTA. Assuming that the transducer complex $\mathbf{1} \cdot \mathrm{Cu}^{2+}$ is equally distributed between the inner and outer leaflets of the vesicle bilayer membranes, the concentration of transducer complex $\mathbf{1} \cdot \mathrm{Cu}^{2+}$ that is exposed to the external EDTA solution is also $5 \mu \mathrm{M}$. The data in Fig. 7 therefore correspond to a tight binding isotherm: at low concentrations of EDTA, every equivalent of EDTA that is added generates active catalyst leading to an increase in the observed rate constant; when one equivalent of EDTA has been added, the maximum concentration of the $1 . \mathrm{Zn}^{2+}$ catalyst is reached, and no further increase in rate constant is observed in the presence of excess EDTA.

A key feature of transmembrane signal transduction mediated by membrane spanning signaling proteins is reversible switching between the OFF and ON states. This enables the system to be reset and respond to subsequent pulses of signaling molecules. The reversibility of the artificial signal transduction process described above was demonstrated by successive additions of EDTA and $\mathrm{CuCl}_{2}$ to the extra-vesicle solution (Fig. 8). Addition of EDTA to a suspension of the vesicles turned $\mathrm{ON}$ the signaling process. Subsequent addition of $\mathrm{CuCl}_{2}$ returned the system to the OFF state by binding to the phenanthroline head group of the transducer. The system was returned to the ON state by further addition of EDTA to the solution to displace the bound copper ions, leading to turnover of remaining unreacted ester $\mathbf{2}$ inside the vesicles. This switching behavior is made possible by the reversible zinc-pyridine oxime binding equilibrium on the inside of the vesicle (Fig. 5). At all times, a small amount of the transducer is not bound to a metal ion at either head group. This species is free to translocate backwards and forwards across the membrane, providing a pathway for equilibration depending on the relative metal ion concentrations on the inside and outside of the vesicle. The mobile transducers can be trapped by copper ions on the outside of the vesicle or zinc ions on the inside of the vesicle, but they are never $100 \%$ bound at either site.

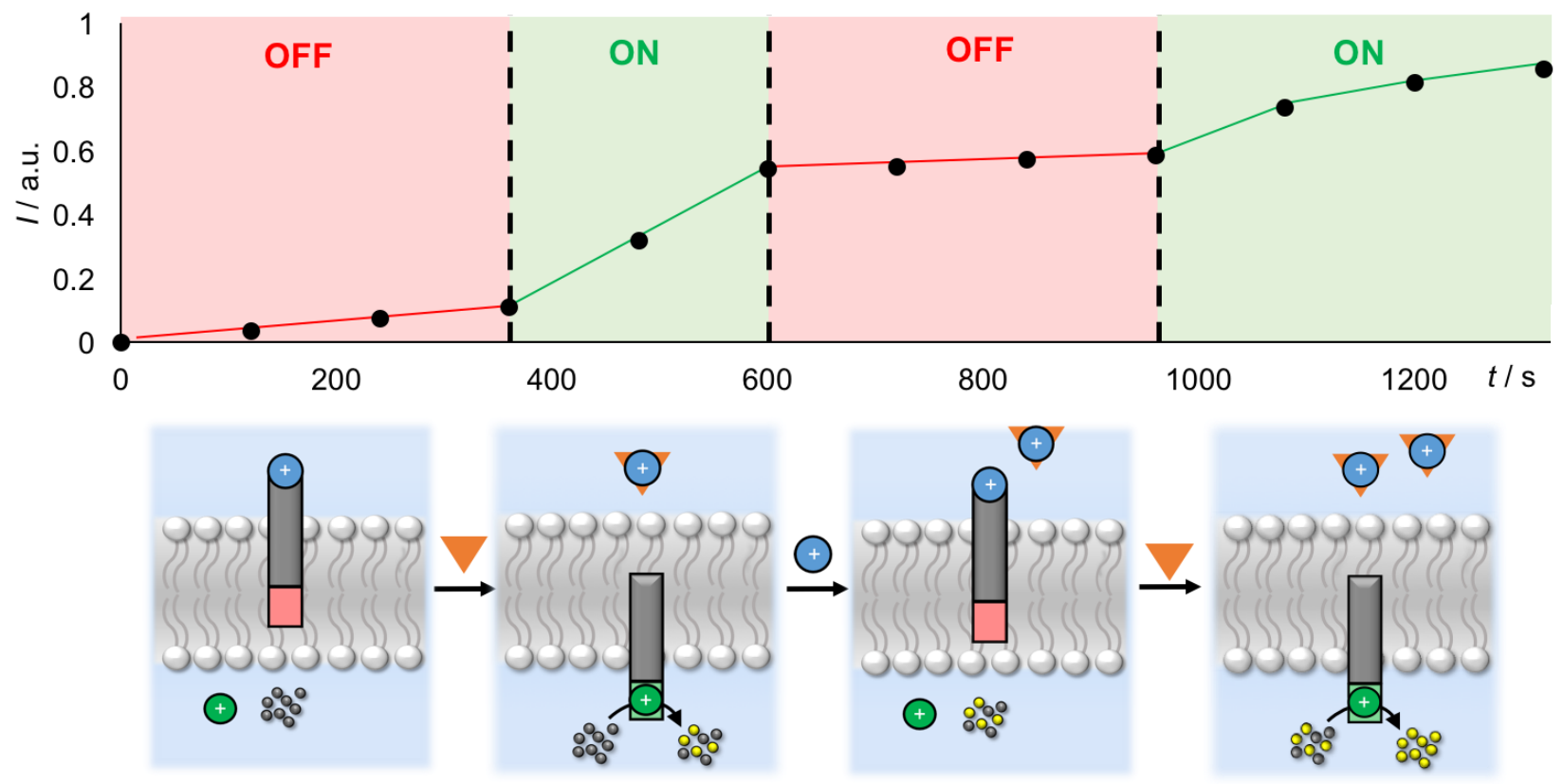

Figure 8. Cycling the external phenanthroline head group between the $\mathrm{Cu}(\mathrm{II})$ complex and free ligand moves transducer 1 backwards and forwards across the membrane, switching the catalyst on the inside of the vesicles OFF and ON. The black data shows the time dependence of the relative fluorescence emission intensity at $510 \mathrm{~nm}$ (exciting at $415 \mathrm{~nm}$ ) during two strokes of a reciprocating cycle: the transduction is repeatedly turned ON by addition of $5 \mu \mathrm{M}$ EDTA (orange triangle) and OFF by addition of $5 \mu \mathrm{M} \mathrm{CuCl}_{2}$ (blue circle) to the external solution. The experimental conditions are the same as Fig. 4. 


\section{CONCLUSIONS}

Host-guest interactions on the outer surface of vesicles have been used to control reversible membrane translocation of a signaling molecule as a mechanism for transmembrane signal transduction and amplification. These findings demonstrate that the membrane translocation mechanism that we reported previously can be generalized to input signals that involve a molecular binding event, analogous to the binding of small molecule signals to membrane signaling proteins. This work will provide the impetus to widen the scope of this methodology to access vesicles responsive to diverse chemical signals, and ultimately provide the basis for developing bio-inspired nanotechnologies capable of interfacing and exchanging information with biological systems.

\section{ASSOCIATED CONTENT}

\section{Supporting Information}

The Supporting Information is available free of charge on the ACS Publications website at DOI: $10.1039 / \mathrm{x} 0 \mathrm{xx} 00000 \mathrm{x}$.

Synthetic procedures, characterization, and additional experimental details (PDF)

\section{AUTHOR INFORMATION}

\section{Corresponding Author}

* Department of Chemistry, University of Cambridge, Lensfield Road, Cambridge CB2 1EW, UK.

E-mail:mj197@cam.ac.uk

\section{ORCID}

* Matthew J. Langton: 0000-0003-1555-3479

Christopher A. Hunter: 0000-0002-5182-1859

Nicholas H. Williams: 0000-0002-4457-422

\section{Notes}

The authors declare no competing financial interests.

\section{ACKNOWLEDGMENT}

We thank the University of Cambridge Oppenheimer Research Fund for an Early Career Research Fellowship (M.J.L).

\section{REFERENCES}

(1) Krauss, G. Biochemistry of Signal Transduction and Regulation, 3rd ed.; Wiley: New York, 2006.
(2) Schlessinger, J. Cell 2000, 103, 211.

(3) Simon, M. I.; Strathmann, M. P.; Gautam, N. Science 1991, 252, 802.

(4) Bennett, I. M.; Farfano, H. M. V.; Bogani, F.; Primak, A.; Liddell, P. A.; Otero, L.; Sereno, L.; Silber, J. J.; Moore, A. L.; Moore, T. A.; Gust, D. Nature 2002, 420, 398.

(5) Sakai, N.; Mareda, J.; Matile, S. Acc. Chem. Res. 2005, 38 (2), 79.

(6) Fyles, T. M. Chem. Soc. Rev. 2007, 36, 335.

(7) Davis, J. T.; Okunola, O.; Quesada, R. Chem. Soc. Rev. 2010, 39, 3843.

(8) Matile, S.; Jentzsch, A. V.; Montenegro, J.; Fin, A. Chem. Soc. Rev. 2011, 40, 2453.

(9) Gale, P. A. Acc. Chem. Res. 2011, 44, 216.

(10) Stanzl, E. G.; Trantow, B. M.; Vargas, J. R.; Wender, P. A. Acc. Chem. Res. 2013, 46, 2944.

(11) Grimaldi, J. J.; Boileau, S.; Lehn, J.-M. Nature 1977, 265, 229.

(12) Barton, P.; Hunter, C. A.; Potter, T. J.; Webb, S. J.; Williams, N. H. Angew. Chem. Int. Ed. 2002, 41, 3878.

(13) Dijkstra, H. P.; Hutchinson, J. J.; Hunter, C. A.; Qin, H.; Tomas, S.; Webb, S. J.; Williams, N. H. Chem. - Eur. J. 2007, 13, 7215.

(14) Bernitzki, K.; Schrader, T. Angew. Chem. Int. Ed. 2009, 48, 8001.

(15) Bernitzki, K.; Maue, M.; Schrader, T. Chem. - Eur. J. 2012, 18, 13412.

(16) De Poli, M.; Zawodny, W.; Quinonero, O.; Lorch, M.; Webb, S. J.; Clayden, J. Science 2016, 352, 575.

(17) Langton, M. J.; Keymeulen, F.; Ciaccia, M.; Williams, N. H.; Hunter, C. A. Nat. Chem. 2016, DOI:10.1038/NChem.2678.

(18) Yatsimirsky, A. K.; Gómez-Tagle, P.; Escalante-Tovar, S.; RuizRamírez, L. Inorganica Chim. Acta 1998, 273, 167.

(19) Wolfbeis, O. S.; Koller, E. Anal. Biochem. 1983, 129, 365.

(20) Clement, N. R.; Gould, J. M. Biochemistry (Mosc.) 1981, 20, 1534.

(21) Wang, P. S. P.; Nguyen, J. B.; Schepartz, A. J. Am. Chem. Soc. 2014, 136, 6810 .

(22) Irving, H.; Mellor, D. H. J. Chem. Soc., 1962, 5222.

(23) MacDonald, R. C.; MacDonald, R. I.; Menco, B. P.; Takeshita, K.; Subbarao, N. K.; Hu, L. R. Biochim. Biophys. Acta 1991, 1061 , 297.

(24) $\log K$ values $\left(\mathrm{M}^{-1}\right)$ for 1:1 metal complexation in water (refs 18 and 22): $\mathrm{Cu} \cdot \mathrm{Phen}=9.0 ; \mathrm{Zn} \cdot \mathrm{Phen}=6.4 ; \mathrm{Zn} \cdot \mathrm{PyO}=4.6 ; \mathrm{Cu} \cdot \mathrm{PyO}$ $=6.7$ (see ESI). Binding of a second equivalent of phenanthroline to $\mathrm{Cu} \cdot \mathrm{Phen}$ is considerably weaker $\left(\log K=5.5 \mathrm{M}^{-1}\right.$, ref 22$)$, and $1: 1$ stoichiometry is expected to dominate under the experimental condition in the absence of EDTA.

(25) Smolarsky, M.; Teitelbaum, D.; Sela, M.; Gitler, C. J. Immunol. Methods 1977, 15, 255.

(26) Anderegg, G. Critical survey of Stability Constants of EDTA complexes, 1st ed.; IUPAC Chemical Data Series; Pergamon Press: Oxford, UK, 1977. 
Controlled

molecular motion

Recognition outside 